\title{
SCA-Based Reconfigurable Access Terminal
}

\author{
Junsik Kim ${ }^{1}$, Sangchul Oh ${ }^{1}$, Eunseon Cho ${ }^{1}$, Namhoon Park ${ }^{1}$, and Nam Kim ${ }^{2}$ \\ ${ }^{1}$ Mobile Telecommunication Research Division \\ Electronics and Telecommunications Research Institute, Daejeon, Korea \\ \{junsik, scoh, escho, nhpark\} @etri.re.kr \\ ${ }^{2}$ Dept. of Comput. \& Commun. Eng., Chung-Buk Nat. Univ., Cheongju, Korea \\ namkim@ehungbuk.ac.kr
}

\begin{abstract}
In this paper, we propose a Reconfigurable Access Terminal (RAT) which is composed of SDR(Software Defined Radio) hardware platform test bed and SCA(Software Communication Architecture) - based software platform. Specifically, we propose a design of the SDR Access Terminal middleware, wireless protocol software component and the procedure of reconfiguration which changes one mode into the other mode. Our RAT system shows that it is capable of changing mode between WiMAX(World Interoperability for Microwave Access) and HSDPA(High Speed Downlink Packet Access). The radio access protocol and application components of RAT are designed through a SCA adapter.
\end{abstract}

Keywords: SCA; SDR, Reconfiguration, Software Component, Middleware.

\section{Introduction}

The SDR, which is a communications device whose functionality is defined in software, has made itself become a key enabling technology in order to realize such a flexible and reconfigurable radio system. Several research works have involved in the development of SDR systems and their efforts focus on hardware design and software framework. However, the current SDR system is insufficient to provide their full potential due to the performance problems. The SCA specification by JTRS(Joint Tactical Radio System) establishes a hardware-independent development framework with baseline requirements for the software definable radios [1]. The SCA has been published to provide a common open architecture that can be used to build a family of radios across multiple domains. The SCA also supports software reusability. Meanwhile, the SCA aims to define a middleware that allows baseband, modulation, protocols modules working together[2][3].

The remainder of this paper is as follows. We provide an overview of the SCA which is a software platform for the RAT and provides a flexible and reconfigurable environment in Section 2. In Section 3, we present the RAT system architecture and functionalities which includes hardware platform and software platform architecture. Then, we describe the procedure of reconfiguration function. Finally, conclusions are drawn. 


\section{SCA Overview}

The SCA defines an Operating Environment (OE) and specifies the services and interfaces that the applications use from the environment. The interfaces are defined by using CORBA IDL and graphical representations are made by using Unified Modeling Language (UML). The OE consists of a Core Framework $(\mathrm{CF})$ that is the essential set for the open interface, a CORBA middleware and a POSIX-based OS. The CF describes the interfaces, their purposes and operations[1]. It provides an abstraction of the underlying software and hardware layers for software application developers. The SCA compatible systems must implement these interfaces. The application components of the SCA divide into two parts, CORBA and Non-CORBA components. The communication between CORBA components and Non-CORBA components is possible through a SCA adapter[4].

\section{Reconfigurable Access Terminal}

We describe our sample RAT platform architecture based on SCA. Fig. 1 describes the reference implementation system architecture for demonstrating SCA-based RAT software download function between WiMAX and HSDPA. On the hardware platform, it is organized by the air interface waveform software components from the software download center using over the air download scheme[2].

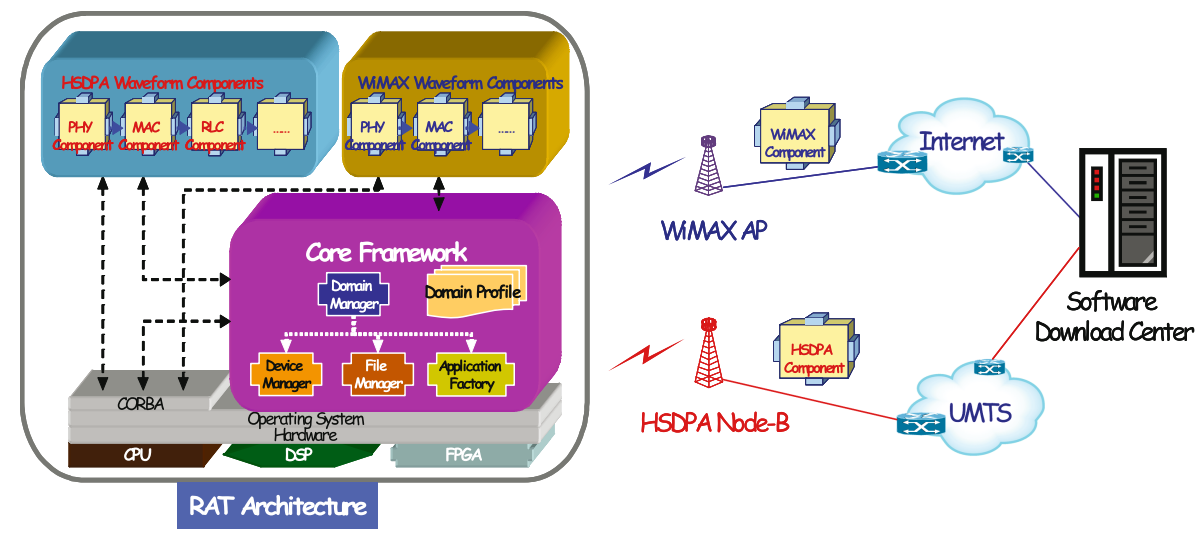

Fig. 1. Reconfigurable Access Terminal

\subsection{RAT Hardware Platform Architecture}

The functional modules on hardware platform are composed of mother board, processor board with GPP(General Purpose Processor), FPGA for base band modem, IF board, RF module and reserved DSP board. As user interface ports, Ether-net port, USE2.0 port, UART port and JTAG for FPGA downloading/debugging are provided. As main control processor, PowerPC embedded in XC2VP30 operates at $150 \mathrm{MHz}$ (Upto $300 \mathrm{MHz}$ is available in Xilinx data sheet). 128 Mbyte FLASH is used for 
program ROM, 128 Mbyte SDRAM is used for program RAM. Two 1Mbit DPRAM (CY7C028) are provided for reserved data memory for MAC hardware. The both of RF and HSDPA modem is under construction, therefore, at the HSDPA mode, the lower transport layer of MAC connected to LAN through a modem simulator and tested.

\subsection{RAT Software Platform Architecture}

The RTOS, CORBA, and SCA CF reside on the main board memory and application components are downloaded from the download center or other auxiliary memory. The radio protocol and application components of the RAT system divide into two parts, CORBA and Non-CORBA components. The communication between CORBA components and Non-CORBA components is possible through the SCA adapters.

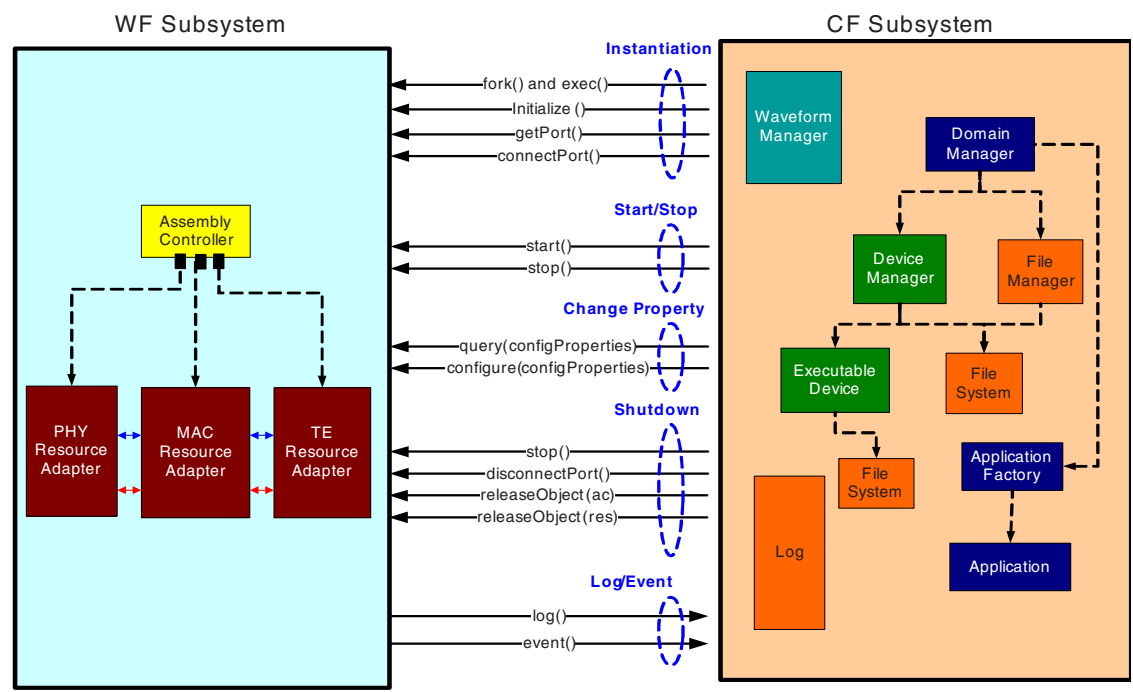

Fig. 2. RAT middleware operation on the Software platform

Fig. 2 shows the middleware operation on the software platform and interfaces between the subsystems of our RAT system. The software platform is specified by two main subsystems which are CF (Core Framework) subsystem and WF (Waveform) subsystem. The CF subsystem, provides the SCA software environment that explained before, makes the efficient operation each manager possible as the middleware which comprises the waveform which is the application managing the wireless access and which it runs. The WF subsystem is comprised of the wireless access protocol and application components, and it is applied in the position of a middleware with the object of a drive. The CF subsystem installs and comprises the wireless access software components on the WF subsystem through a command including the start / stop, the change property for the parameter delivery, a shutdown, the history log / event for message passing, and etc. and runs. 


\section{Reconfigure Procedure}

In previous section, we presented the hardware and software platform of the RAT system. Each platform has the proper structure to reconfigure the system. In this section, we describe the mode change function as the core function of the SDR. The mode change function is initiated from the RATM which is software block, and responsible for the management of the RAT. As mentioned above, the RAT can provide the service of two modes i.e. WiMAX and HSDPA independently. When the RAT is currently on service with the WiMAX mode, the user (or operator) via the RATM wants to change the HSDPA mode. When the RATM decides to change the mode, it sends the change mode request with a HSDPA software package to the MC which is software block and responsible for the mode control of the RAT, and it unpacks and installs the HSDPA software package into the specified device modules. The $\mathrm{MC}$ sends the change mode request to the device modules. Once receiving the change mode request, the device modules let the WiMAX software modules stop and shutdown. If the change mode procedure of the WiMAX software modules is complete, the $\mathrm{MC}$ sends the reconfigure request to the component update unit. After the update completion, the MC sends the execution request to the device module. Once receiving the execution request, the device modules let the software modules of the HSDPA run. If the entire change mode procedure is complete, the MC responses the RATM.

\section{Conclusion}

This paper represents a Reconfigurable Access Terminal developed on the basis of the SCA standard architecture and it is capable of providing the WiMAX and the HSDPA service. The RAT currently adopted an interim architecture using a SCA adaptor. However, if there are more advances in the SCA technology as well as the SDR hardware, the architecture of the RAT will be upgraded for the system performance. Specifically, we designed SCA-based RAT software platform and illustrated the procedure of reconfiguration which changes one mode into the other mode. The research of reducing the reconfiguration time, FPGA componentization, and software modem improvement should be studied later.

\section{References}

1. JTRS website, http://www.jtrs.army.mil

2. SDR Forum website, http://www.sdrforum.org

3. Saehwa Kim, Jamison Masse, Seongsoo Hong, and Naehyuck Chang : SCA-based Component Framework for Software Defined Radio, Proceedings of the IEEE Workshop on Software Technologies for Future Embedded Systems 2003, May 15-16, (2003) 3-6

4. EunSeon Cho, ChangKi Kim, YeonSeung Shin, and JinUp Kim : SCA-based multi-LAN application development, Proceedings of VTC2004-Fall, 26-29 Sept. (2004) 1978 - 1982 